\title{
Nitrogen Matters:
}

\section{The Difference Between PANH and PAH Formation}

\author{
Jordy Bouwman, ${ }^{1, *}$ Andras Bodi, ${ }^{2}$ and Patrick Hemberger ${ }^{2}$ \\ ${ }^{1}$ Sackler Laboratory for Astrophysics, Leiden Observatory, Leiden University, P.O. Box 9513, \\ NL 2300 RA Leiden, The Netherlands \\ ${ }^{2}$ Laboratory for Synchrotron Radiation and Femtochemistry, Paul Scherrer Institute, 5232 \\ Villigen, Switzerland \\ e-mail: bouwman@strw.leidenuniv.nl
}

\begin{abstract}
A solid understanding of and a reliable model for the formation pathways of polycyclic aromatic (nitrogen containing) hydrocarbons $(\mathrm{PA}(\mathrm{N}) \mathrm{Hs})$ is needed to account for their abundance in the interstellar medium and in Titan's atmosphere and to mitigate the emission of these carcinogens in our terrestrial environment. We investigate the phenyl + acrylonitrile reaction mechanism between 600 and $1200 \mathrm{~K}$ in a hot microreactor. Radical intermediates $\left(\mathrm{C}_{9} \mathrm{H}_{8} \mathrm{~N} \cdot\right)$, formed by addition, and closed-shell $\mathrm{C}_{9} \mathrm{H}_{7} \mathrm{~N}$ products, formed by subsequent hydrogen elimination, are isomer-selectively identified using photoion mass-selected threshold photoelectron spectroscopy in conjunction with Franck-Condon simulations. Although quinoline is the most stable product, the calculated potential energy surface and a kinetic model confirm that the reaction is kinetically controlled and yields four open-chain isomers instead. The absence of quinoline is in stark contrast with the isoelectronic phenyl + vinylacetylene reaction that produces naphthalene. Ab initio calculations suggest that this change is brought about by the stability of the nitrile group, which inhibits ring formation. Therefore, it is unlikely that nitrile precursors form nitrogen-containing rings, which calls for alternative pathways by which nitrogen atoms can be incorporated in aromatic systems to explain their presence in the ISM and Titan's atmosphere.
\end{abstract}




\section{Introduction}

Polyaromatic hydrocarbons (PAHs) and, presumably, their nitrogen containing analogues (PANHs) are abundant in the Interstellar Medium (ISM) as evidenced by their characteristic midinfrared bands. ${ }^{1-4}$ Up to $15 \%$ of the total cosmic carbon is locked up in these species, underlining their interstellar importance. They are also present in planetary atmospheres, such as that of planet Earth and of Saturn's largest moon, Titan. Terrestrially, PA(N)Hs are emitted as byproducts of combustion reactions and are considered to be harmful carcinogenic pollutants. ${ }^{5-7}$ In Titan's atmosphere, the presence of $\mathrm{PA}(\mathrm{N}) \mathrm{Hs}$ has been derived from mass spectrometric and spectroscopic data ${ }^{8-10}$ They are thought to be formed from traces of hydrocarbons (up to $2 \%$, with methane being the dominant species) in the predominantly $(\approx 98 \%)$ nitrogen containing atmosphere. ${ }^{11-13}$

The formation of PAHs in the aforementioned, widely different environments has been a source of debate over the past decades. Different chemical mechanisms have been proposed for the formation of homocyclic aromatics, for instance the hydrogen abstraction carbon addition (HACA) mechanism, and the phenyl addition mechanism among them. ${ }^{14-19}$ A mechanism involving reactions between (poly)cyclic aromatic radicals (such as phenyl or naphthyl) and vinylacetylene has also been proposed as an efficient pathway to form PAHs. ${ }^{20-22}$ It was recently shown that the phenyl + vinylacetylene reaction indeed yields the polyaromatic naphthalene among other products. $^{23}$ The formation of $\mathrm{N}$-substituted analogs is much less understood and only few reactions have been investigated so far that could potentially yield heterocyclic molecules. For example,

Parker et al. reported a ring growth step connecting pyridine with quinoline, ${ }^{24,25}$ which explains PANH growth, but not the introduction of nitrogen into the PAH scaffold. Unveiling the formation pathways of PANHs helps mitigate the emissions of these carcinogens in our terrestrial 
environment and understand their formation and large abundance in the interstellar medium and in Titan's atmosphere.

In this work, we focus on the possible formation of $\mathrm{N}$-substituted polyaromatics from the reaction between acrylonitrile (vinyl cyanide, $\left.\mathrm{C}_{3} \mathrm{H}_{3} \mathrm{~N}\right)$ and phenyl radicals $\left(\mathrm{C}_{6} \mathrm{H}_{5} \bullet\right.$ ) in a hot microreactor as a function of the temperature. This system is isoelectronic with the recently studied phenyl + vinylacetylene reaction to naphthalene and may offer a novel pathway for the incorporation of nitrogen in polyaromatic molecules:

$$
\mathrm{C}_{6} \mathrm{H}_{5} \bullet+\mathrm{C}_{3} \mathrm{H}_{3} \mathrm{~N} \rightarrow \mathrm{C}_{9} \mathrm{H}_{8} \mathrm{~N} \bullet+\mathrm{C}_{9} \mathrm{H}_{7} \mathrm{~N}+\mathrm{H} \bullet
$$

Intermediates and reaction products leaving the reactor expand into high vacuum to form a molecular beam and are analyzed using photoelectron photoion coincidence (PEPICO) spectroscopy. ${ }^{26,27}$ Photoion mass-selected threshold photoelectron spectra (ms-TPES) allow for isomer-selective detection, when combined with Franck-Condon modeling of the band profiles and intensities. This approach has been used in the identification and characterization of elusive reactive intermediates, ${ }^{28}$ which help unveil complex reaction mechanisms in diverse reactive environments. ${ }^{29-31} A b$ initio calculations and kinetic modeling are performed to explore the potential energy surface and rationalize the experimental data. ${ }^{32}$ Contrary to our expectations, based primarily on naphthalene formation in the analogous phenyl + vinylacetylene reaction, ${ }^{23}$ we did not detect quinoline and argue that it may only be a minor reaction product. We point out the extraordinary stability of the nitrile group as an underlying reason inhibiting ring formation and raise the possibility that, analogously to the yet unknown quinoline formation mechanism, further reactive pathways could yield PAHs, as well. 


\section{Methods}

\subsection{Experimental}

The experiments have been performed in a pyrolysis reactor coupled to the CRF-PEPICO double imaging photoelectron photoion coincidence $\left(i^{2} \mathrm{PEPICO}\right)$ endstation at the Vacuum Ultraviolet beamline of the Swiss Light Source (SLS) at Paul Scherer Institute (PSI). The PEPICO system and beamline have been discussed in detail in the literature ${ }^{33-35}$ and only a summary is presented here.

Nitrosobenzene is used as a phenyl radical precursor, as has been extensively reported in the literature ${ }^{16,36}$ and acrylonitrile is introduced as a reactant (see Figure 1 for molecular structures). Both were purchased from Sigma-Aldrich $\left(\geq 97 \%\right.$ and $\geq 99 \%$ for $\mathrm{C}_{6} \mathrm{H}_{5} \mathrm{NO}$ and $\mathrm{C}_{3} \mathrm{H}_{3} \mathrm{~N}$, respectively) and used without further purification. Two separate bubblers are connected in series and contain nitrosobenzene and acrylonitrile. An Ar flow of $70 \mathrm{sccm}$ at a pressure of 0.5 bar picks up the vapor of both species, yielding a mixture of $\approx 2 \%$ nitrosobenzene and $20 \%$ acrylonitrile in argon. Additional control measurements on $2.5 \% \mathrm{C}_{6} \mathrm{H}_{5} \mathrm{NO}$ in argon and $20 \%$ acrylonitrile in argon have been performed to confirm the products of the phenyl-acrylonitrile reaction. The gas mixture is expanded through a $100 \mu \mathrm{m}$ pinhole into the pyrolysis tube reactor, which is a $\approx 3 \mathrm{~cm}$ long $1 \mathrm{~mm}$ internal diameter resistively heated $\mathrm{SiC}$ tube. The surface temperature of the reactor was measured by a Type $\mathrm{C}$ thermocouple, which is expected to represent the gas temperature inside the reactor to within $100 \mathrm{~K}$. We have estimated the temperature, pressure and residence time in the reactor based on the extensive modeling study of Guan et al. ${ }^{37}$ to be $500-1000 \mathrm{~K}, 10-40$ mbar and ca. 100 $\mu \mathrm{s}$. The reactor is placed in the source vacuum chamber, where the pressure was $4 \times 10^{-5}$ mbar during measurements. The molecular beam exiting the pyrolysis reactor passes through a $1 \mathrm{~mm}$ diameter skimmer into the detection chamber, which is kept at a pressure of $1 \times 10^{-6}$ mbar. 

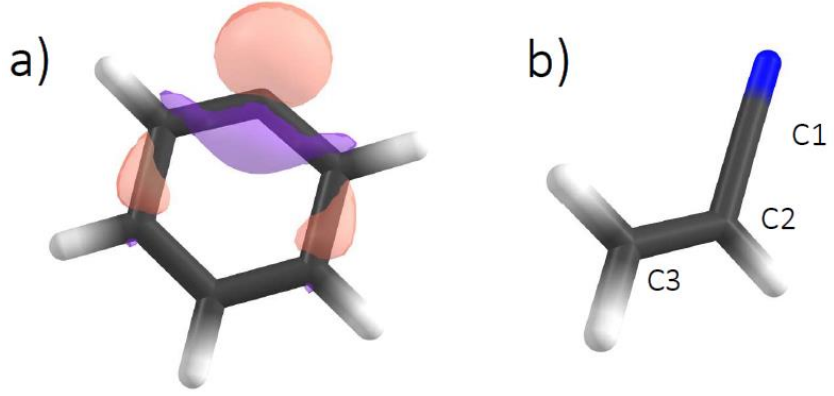

Figure 1. (a) Molecular structure of the phenyl radical shown together with a rendering of the highest occupied molecular orbital (HOMO). (b) Acrylonitrile with labeling of the carbon atoms.

Synchrotron VUV radiation is generated using a bending magnet, dispersed on a 150 grooves/mm grating and focused at the exit slit, resulting in an energy resolution $5 \mathrm{meV}$ at $8 \mathrm{eV}$. The VUV beam passes through a $\mathrm{MgF}_{2}$ window to remove contributions by higher order radiation, and ionizes the sample in the molecular beam at a $4 \times 2 \mathrm{~mm}^{2}$ spot size. The resulting photoelectrons and -ions are extracted in opposite direction by a constant $250 \mathrm{~V} / \mathrm{cm}$ field. The electrons are velocity map imaged on a RoentDek delay line detector and also serve as the start signal for the time-of-flight (TOF) measurement of the coincident ion. The position of the electron on the detector reveals information on its lateral velocity with threshold electrons imaged onto the center of the detector. The ions mass analysis is carried out in a Wiley-McLaren TOF tube and the ions are also detected on a RoentDek delay line detector.

The photon energy was tuned between 7.2 to $10.0 \mathrm{eV}$ in steps of $0.025 \mathrm{eV}$ and all electron and ion events were recorded in a multiple-start/multiple-stop setup. ${ }^{38}$ Mass spectra are plotted by finding delayed coincidences between all electrons and ions. Threshold, low kinetic energy $(<10$ $\mathrm{meV}$ ) photoelectrons were discriminated for ${ }^{39,40}$ and selectively allowed as start signal to obtain photoion mass-selected threshold photoelectron spectra by plotting the intensity of a mass channel as a function of VUV energy. 


\subsection{Computational}

Quantum chemical computations have been performed using the Gaussian09 and Gaussian16 suites of programs ${ }^{41}$ to explore the $\mathrm{C}_{9} \mathrm{H}_{8} \mathrm{~N}$ potential energy surface (PES) and compare it with that of $\mathrm{C}_{10} \mathrm{H}_{9}$. Molecular coordinates have been scanned at the B3LYP/6-311++G(d,p) level of theory to locate stationary points and transition states. Transition states with a critical vibration that did not obviously correspond to the assumed reaction coordinate have been further investigated using intrinsic reaction coordinate (IRC) scans to confirm that they connect the proper stationary points on the PES. Stationary points and transition states are subsequently re-evaluated at the CBS-QB3 level of theory to obtain more accurate energies. ${ }^{42,43}$

Threshold photoelectron spectra of possible intermediates and products have been simulated employing the eZspectrum ${ }^{44}$ and Gaussian16 software packages to allow for isomer-selective assignment. ${ }^{31,32,45,46}$ Franck-Condon factors have been calculated at 400 to $500 \mathrm{~K}$, which are typical final temperatures for species produced in hot pyrolysis reactors. ${ }^{28,46}$ The stick spectra were subsequently convoluted with a $30-50 \mathrm{meV}$ broad Gaussian function to account for the rotational envelope and to facilitate comparison with the experimental spectra. The adiabatic ionization energy is determined from the CBS-QB3 calculated energies of the neutral and ionic product species. $^{42,43}$

Harmonic vibrational frequencies obtained for the stationary points also allow us to calculate Rice-Ramsperger-Kassel-Marcus (RRKM) unimolecular hydrogen atom loss rates as a function

of internal energy. ${ }^{47}$ The rate curves can then be used to estimate the lifetime of an adduct with respect to $\mathrm{m} / z 129$ closed-shell product formation and to rationalize the missing quinoline signal. 


\section{Results and discussion}

\subsection{Mass spectrometry}

A time-of-flight mass spectrum of $2.5 \%$ nitrosobenzene with $20 \%$ acrylonitrile in argon pyrolyzed at $690 \mathrm{~K}$ and recorded at a photon energy of $10 \mathrm{eV}$ is shown in Figure 2. The ionization energies relevant to the discussion of these main products are listed in Table 1.

The unpyrolyzed phenyl radical precursor, nitrosobenzene, is observed at $\mathrm{m} / \mathrm{z}, 107$. Further peaks appear only upon pyrolysis. The products formed from the thermal decomposition of nitrosobenzene, phenyl and nitric oxide, appear at $\mathrm{m} / \mathrm{z} 77$ and 30. Acrylonitrile is not observed in the mass spectrum, as it is recorded below its ionization threshold of $10.91 \mathrm{eV}$. The main signals at $m / z, 129$ and 130 originate from bimolecular adduct formation and subsequent, predominantly unimolecular, hydrogen atom loss according to reaction (1). The phenyl radical is an efficient hydrogen scavenger, which explains the peak at $m / z 78$ corresponding to benzene. While a detailed discussion of the possible side reactions is beyond our scope, most of the minor product peaks could be assigned and are discussed in detail in the electronic supplementary information (ESI). 


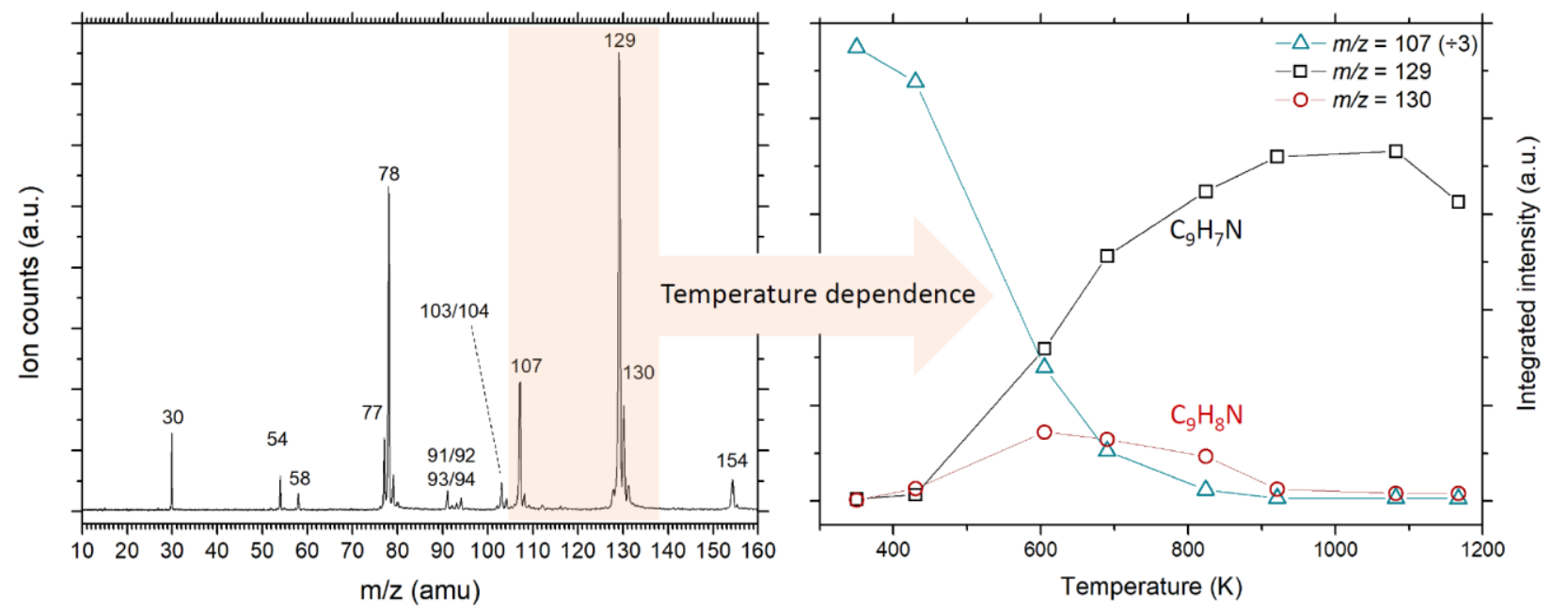

Figure 2. (left) All-electron time-of-flight mass spectrum of $2.5 \%$ nitrosobenzene and $20 \%$ acrylonitrile in argon, recorded at a photon energy of $10 \mathrm{eV}$ and a pyrolysis reactor temperature of $690 \mathrm{~K}$. The species are assigned in Table 1. (right) Evolution of the integrated $\mathrm{m} / \mathrm{z} \mathrm{107,129}$ and 130 signal recorded at $9 \mathrm{eV}$ as a function of pyrolysis temperature.

Table 1: List of ionization energies of species that are relevant for this work. The asterisk (*) marks a computed adiabatic ionization energy reported in this work.

\begin{tabular}{|c|cc|c|c|}
\hline Species & $m / z$ & name & IP $(\mathrm{eV})$ & Ref. \\
\hline $\mathrm{NO}$ & 30 & Nitric oxide & 9.26 & 48 \\
\hline $\mathrm{C}_{3} \mathrm{H}_{4} \mathrm{~N}$ & 54 & 1-Cyanoethyl radical & 9.28 & $*$ \\
\hline $\mathrm{C}_{3} \mathrm{H}_{3} \mathrm{~N}$ & 55 & Acrylonitrile & 10.91 & 49 \\
\hline $\mathrm{C}_{6} \mathrm{H}_{5} \mathrm{NO}$ & 107 & Nitrosobenzene & 8 & 50 \\
\hline $\mathrm{C}_{6} \mathrm{H}_{5}$ & 77 & Phenyl radical & 8.67 & 51 \\
\hline $\mathrm{C}_{6} \mathrm{H}_{6}$ & 78 & Benzene & 9.24 & 52 \\
\hline $\mathrm{C}_{6} \mathrm{H}_{5} \mathrm{CN}$ & 103 & Cyanobenzene & 9.729 & 53 \\
\hline $\mathrm{C}_{6} \mathrm{H}_{5} \mathrm{CHCH}_{2}$ & 104 & Styrene & 8.464 & 54 \\
\hline $\mathrm{C}_{9} \mathrm{H}_{7} \mathrm{~N}$ & 129 & Z-3-phenylacrylonitrile [P1] & 8.90 & $*$ \\
& & E-3-phenylacrylonitrile [P2] & 8.91 & $*$ \\
& & ortho-cyanostyrene [P3] & 8.98 & $*$ \\
& & 2-phenylacrylonitrile [P4] & 9.03 & $*$ \\
& & Quinoline [P5] & 8.61 & 55 \\
\hline $\mathrm{C}_{12} \mathrm{H}_{10}$ & 154 & Biphenyl & 8.20 & 56 \\
\hline
\end{tabular}


The most intense signals are detected at $m / z, 130$ and 129, which are the products of the phenyl + acrylonitrile addition-elimination reaction (1), and exhibit peculiar temperature dependence. A detailed view on the formation of these two products ionized at a photon energy of $9 \mathrm{eV}$ is shown in the right panel of Figure 2, where the (scaled) integrated ion intensities of the phenyl precursor (nitrosobenzene) and the two most intense product species are shown as a function of reactor temperature. This photon energy is chosen as it is sufficient to ionize both reaction products (vide infra), yet low enough to suppress dissociative ionization. Both products grow in as soon as the radical is formed via pyrolysis of the radical precursor according to the following reactions:

$$
\begin{aligned}
& \mathrm{C}_{6} \mathrm{H}_{5} \mathrm{NO} \stackrel{\Delta}{\rightarrow} \mathrm{C}_{6} \mathrm{H}_{5} \bullet+\mathrm{NO} \\
& \mathrm{C}_{3} \mathrm{H}_{3} \mathrm{~N}+\mathrm{C}_{6} \mathrm{H}_{5} \bullet \rightarrow \mathrm{C}_{9} \mathrm{H}_{8} \mathrm{~N} \bullet \\
& \mathrm{C}_{9} \mathrm{H}_{8} \mathrm{~N} \bullet \rightarrow \mathrm{C}_{9} \mathrm{H}_{7} \mathrm{~N}+\mathrm{H} \bullet
\end{aligned}
$$

The $\mathrm{C}_{9} \mathrm{H}_{8} \mathrm{~N} \bullet$ adduct in reaction $(3)(\mathrm{m} / z, 130)$ is only observed at low reactor temperatures and loses a hydrogen atom as the temperature is increased to generate closed shell $\mathrm{C}_{9} \mathrm{H}_{7} \mathrm{~N}$ species (4).

\subsection{Intermediate and product identification}

Photoion mass selected threshold photoelectron spectra (ms-TPES) have been recorded by scanning the photon energy and considering only photoelectrons with less than $10 \mathrm{meV}$ kinetic energy together with the coincident ion of the chosen $\mathrm{m} / \mathrm{z}$ from the same ionization event. The structure of the band profiles correspond to vibrational transitions from the neutral into the ionic state and Franck-Condon simulations can thus be used to identify the reactive intermediates and stable products. The identification of minor and side products is described in the ESI. 

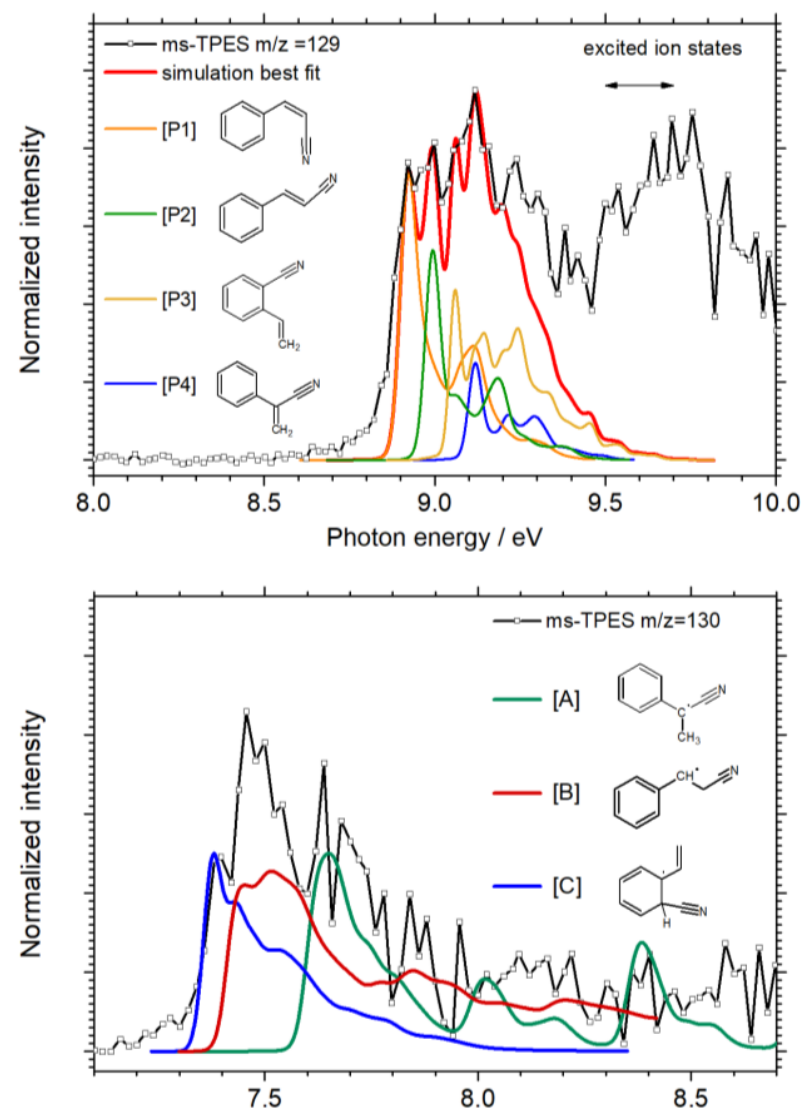

Figure 3: Photoion mass selected threshold ionization spectra of the products with $\mathrm{m} / \mathrm{z} 129$ (top) and the radical adduct with $\mathrm{m} / \mathrm{z} 130$ (bottom) formed from the reaction between phenyl and acrylonitrile, shown together with Franck-Condon simulations Top: The $m / z, 129$ TPE spectrum can be modeled by Z-3-phenylacrylonitrile ([P1]), E-3-phenylacrylonitrile ([P2]), orthocyanostyrene ([P3]) and 2-phenyl acrylonitrile ([P4]). Bottom: Simulated TPE spectra of the potential radical contributors $\alpha$-methyl- $\alpha$-cyano-benzyl radical ([A], green curve), 2-cyano-1phenylethyl radical ([B], red curve) and 6-cyano-1-ethenylcyclohexa-2,4-dien-1-yl ([C], blue curve) are plotted onto the $m / z 130$ spectrum.

\subsection{1 $\mathrm{m} / \mathrm{z} 129$ closed shell reaction product}

The ms-TPES of the $m / z 129$ product recorded at $1167 \mathrm{~K}$ is shown in the top panel of Figure 3 . A total of 17 product isomers have been considered as potential contributors to the TPE spectrum, which are listed in the ESI. The best fit to the data is also shown in Error! Reference source not found. and consists of a superposition of simulated TPES of four products, namely, 2-phenyl 
acrylonitrile, E-3-phenylacrylonitrile, Z-3-phenylacrylonitrile and ortho-cyanostyrene. Quinoline has a sharp ionization threshold at $8.61 \mathrm{eV}$ and a broad vibrational band at $8.70 \mathrm{eV},{ }^{55}$ i.e., below the onset of the ms-TPE spectrum recorded in this study. Therefore, quinoline may only be present in the reaction mixture below the PEPICO detection limit, if at all. While the spectral fit yields the fractional contribution of each isomer, we do not report branching ratios because absolute ionization cross sections are lacking. A mismatch between the measured and simulated $\mathrm{m} / \mathrm{z}, 129$ TPES is apparent at photon energies exceeding $9.4 \mathrm{eV}$. This can be attributed to ionization into higher lying electronic states of 2-phenyl acrylonitrile, $E$ - and Z-3-phenylacrylonitrile, and orthocyanostyrene cations, rather than contributions by other isomers. Indeed, TD-DFT calculations revealed vertical excitation energies of all four isomers between 9.5 and $9.7 \mathrm{eV}$.

\subsection{2 $\mathrm{m} / \mathrm{z} 130$ open shell adduct}

An experimental ms-TPE spectrum of the radical intermediate at $\mathrm{m} / \mathrm{z} 130$, recorded at a reactor temperature of $630 \mathrm{~K}$ is shown in the bottom panel of Figure 3. This temperature is chosen as the intensity of the radical adduct reaches a maximum in the mass spectrum (Figure 2). According to CBS-QB3 ionization energies (see ESI), up to eight different radicals may be the carrier of the msTPES out of 40 evaluated isomers. In view of the calculated reaction pathways (vide infra) and observed closed shell products, we assign the most abundant features of the TPES to $\alpha$-methyla-cyano-benzyl radical (green curve), 6-cyano-1-ethenylcyclohexa-2,4-dien-1-yl (blue curve) and 2-cyano-1-phenylethyl radical (red curve). The latter undergoes a moderate change in geometry upon ionization with an out of plane (phenyl) rotation of the $\mathrm{CH}_{2}-\mathrm{CN}$ group, leading to a broad and fairly unresolved photoelectron band. Due to the hardly resolved ms-TPE spectrum and featureless calculated spectra, we have not fitted the sum of the individual simulations to the experimental spectrum. However, from spectroscopic point of view, the individual isomers of the 
radical adducts $(\mathrm{m} / \mathrm{z}, 130)$ and products $(\mathrm{m} / \mathrm{z}, 129)$ of the acrylonitrile + phenyl reaction can be assigned and the presence of quinoline can clearly be ruled out.

\subsection{Reaction mechanism and kinetic modeling}

The absence of quinoline is in stark contrast to the isoelectronic phenyl + vinylacetylene reaction, which yields the bicyclic naphthalene species along with open chain products. Thus, the $\mathrm{C}_{9} \mathrm{H}_{8} \mathrm{~N}$ potential energy surface (PES) has been explored to reveal the underlying reaction mechanism of the phenyl + acrylonitrile reaction and to explain the absence of quinoline. A summary of the rate limiting transition states and main products is shown in Figure 4, while the full PES results are presented in the ESI.

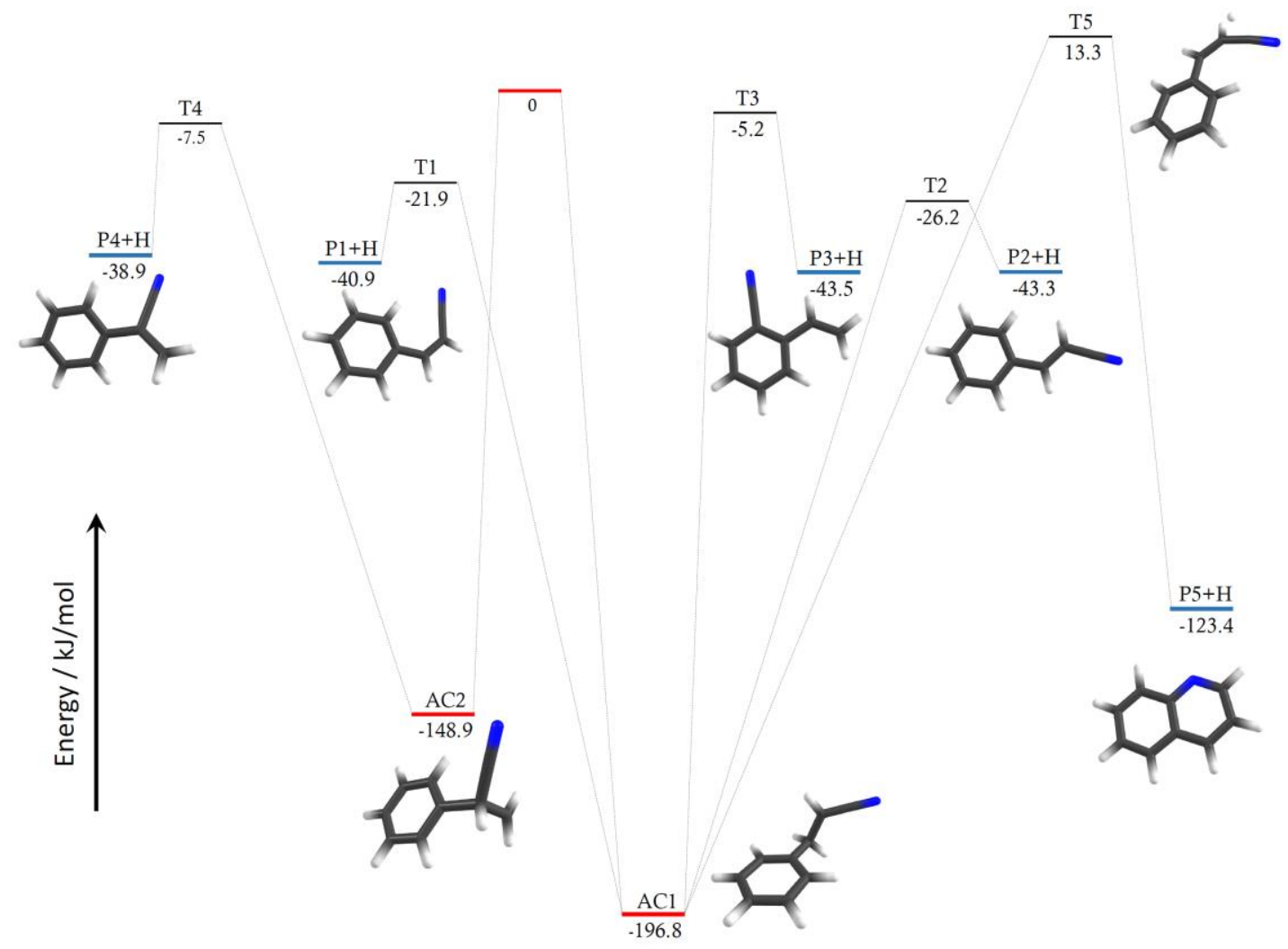

Figure 4: Summary of the $\mathrm{C}_{9} \mathrm{H}_{8} \mathrm{~N}$ potential energy surface showing only the rate limiting transition states. The rate limiting transition state to quinoline, T5, involves a hydrogen migration and is explicitly shown. 
Addition complex 1 (AC1) is formed without an entrance barrier. From AC1, four product channels are available, of which three involve rate limiting transition states that lie below the entrance channel energy. Z-3-phenylacrylonitrile (P1) is formed over rate limiting transition state (T1) located at $-21.9 \mathrm{~kJ} / \mathrm{mol}$, while the channel to yield $E$-3-phenylacrylonitrile (P2) possesses a slightly lower lying barrier (T2) at $-26.2 \mathrm{~kJ} / \mathrm{mol}$. Transition state (T3) to ortho-cyanostyrene (P3) is located at $-5.2 \mathrm{~kJ} / \mathrm{mol}$ relative to the starting material. Most notably, the lowest energy reaction channel to quinoline (P5) starting from AC1 requires crossing of a rate limiting transition state (T5) at $+13.3 \mathrm{~kJ} / \mathrm{mol}$ with respect to the entrance channel. An alternative pathway to 2-phenyl acrylonitrile (P4) starts with the barrierless formation of addition complex 2 (AC2), followed by a submerged barrier (T4) at $-7.5 \mathrm{~kJ} / \mathrm{mol}$.

The PES in Figure 4 indicates that the pathway to quinoline is kinetically hindered compared to the formation of the open chain isomers. We also constructed an RRKM model of the H-atom elimination from the AC1 intermediate, ${ }^{47}$ as discussed in the ESI. The model predicts that the lifetime of AC1 is on the order of $10 \mu \mathrm{s}$ at the experimental temperatures, i.e., lower than the residence time in the reactor. AC1 is predicted to form $\mathbf{P 2}$ and $\mathbf{P 1}$ predominantly, and the RRKM branching ratio to quinoline is less than $1 \%$ up to $400 \mathrm{~kJ} / \mathrm{mol}$ internal energy.

\subsection{Difference between PANH and PAH formation}

We found that the phenyl + acrylonitrile reaction does not lead to the thermodynamically most stable isomer, quinoline, which is in stark contrast to the isoelectronic phenyl + vinylacetylene reaction yielding $43.5 \%$ naphthalene. ${ }^{23}$ To reveal the differences between the two mechanisms, we performed additional potential energy surface scans on both systems, which are shown in the ESI. Here we focus only on the pathway that reveals the most pronounced difference between the two 
isoelectronic reactions and the PES is shown in Figure 5. It is important to note here that the pathway to quinoline shown in Figure 5 is not the lowest energy pathway as seen in Figure 4, but rather the equivalent of the pathway to naphthalene with the lowest rate limiting barrier.

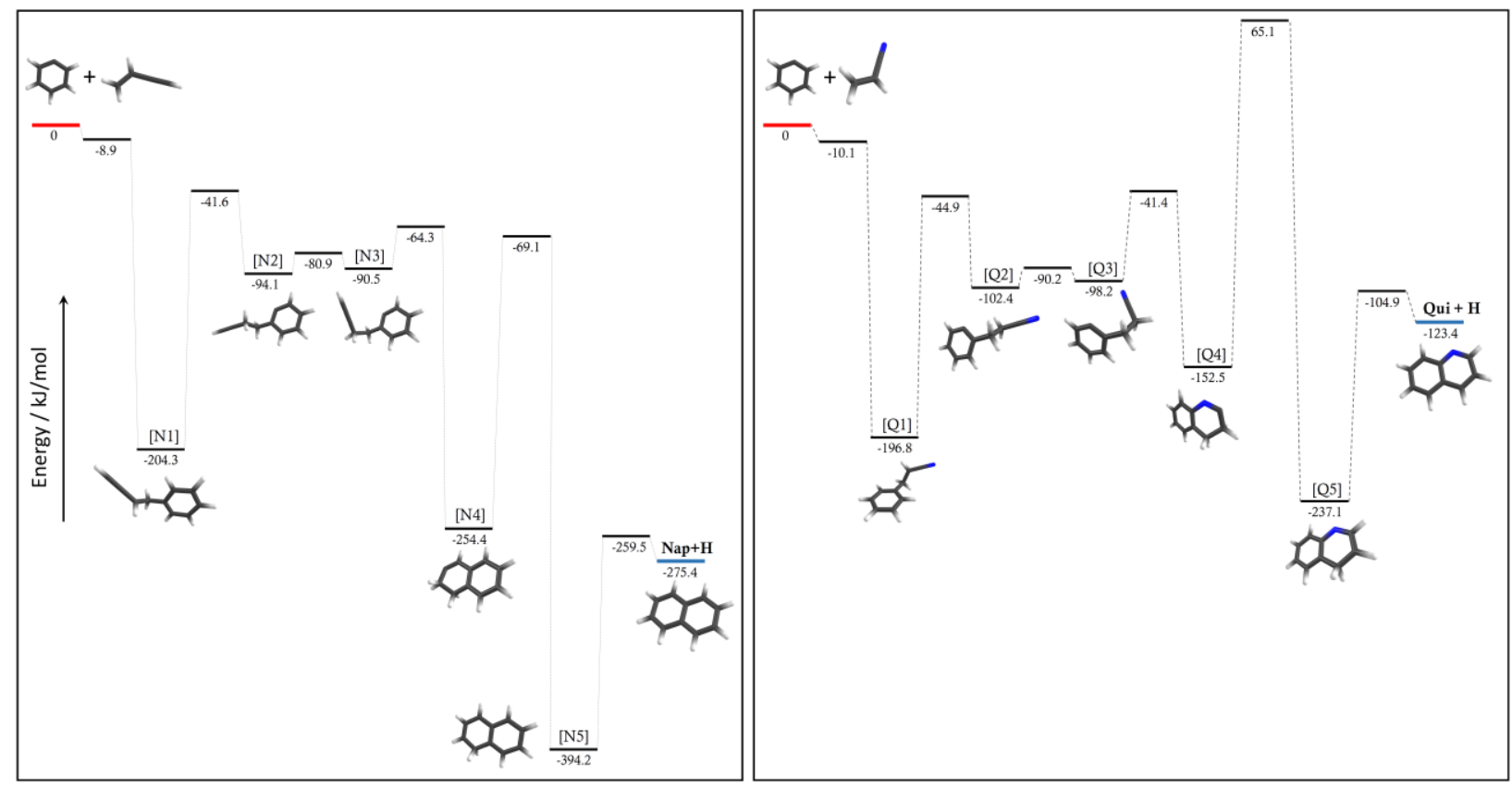

Figure 5: Comparison between naphthalene and quinoline formation mechanisms. Both potential energy surfaces show CBS-QB3 calculated energies relative to their respective reactants. Transition states are available in the ESI.

A clear difference between naphthalene and quinoline formation is apparent from Figure 5. For the case of quinoline, a rate limiting transition state for this transfer is found at $+65.1 \mathrm{~kJ} / \mathrm{mol}$, while this step is well below the entrance channel $(-69.1 \mathrm{~kJ} / \mathrm{mol})$ for naphthalene. The potential energy curves are quite similar up to the ring closure ( $\mathbf{N 3}$ vs. Q3), as well as afterwards, past $\mathbf{N 4}$ and Q4. However, the reaction is less exothermic by about $150 \mathrm{~kJ} / \mathrm{mol}$ in the case of quinoline, and this difference manifests itself first in the ring closure transition state. In order to localize the driving force behind this shift, we have calculated saturation transfer reaction energies using the 
CBS-QB3 composite method, in which a nitrogen atom is exchanged for a $\mathrm{CH}$ group with methane and ammonia as reaction partners:

$$
\mathrm{R}(\mathrm{N})+\mathrm{CH}_{4} \rightarrow \mathrm{R}(\mathrm{CH})+\mathrm{NH}_{3}
$$

In most cases, reaction energies are small, indicating a large similarity in the electronic structure and energetics of the $\mathrm{N}$ and $\mathrm{CH}$ containing species. This applies not only to methylamine/ethane $(-32 \mathrm{~kJ} / \mathrm{mol})$ and pyridine/benzene $(-23 \mathrm{~kJ} / \mathrm{mol})$, but also to quinoline/naphthalene $(-18 \mathrm{~kJ} / \mathrm{mol})$, which means that the difference in energetics is not on the product, but probably on the reactant side in our case. As a matter of fact, when a $\mathrm{C} \equiv \mathrm{N}$ group is exchanged for $\mathrm{C} \equiv \mathrm{CH}$, as in acrylonitrile/vinylacetylene or hydrogen cyanide/acetylene, the reaction energy jumps to 134 or $131 \mathrm{~kJ} / \mathrm{mol}$, respectively. In other words, the ethynyl group is much more readily saturated than the nitrile group, which stabilizes acrylonitrile with respect to vinylacetylene when it comes to ring formation. Although a lower energy pathway opens up in the phenyl + acrylonitrile reaction over TS5 to yield quinoline, it still is too high in energy to have a measurable branching ratio. Therefore, in the absence of reaction paths with only submerged barriers, it appears unlikely that a nitrile group will contribute to ring formation or expansion reactions. More saturated nitrogen compounds are more likely to do so, and such reaction pathways or, alternatively, ion chemistry may play a dominant role leading to PANHs in the interstellar medium and Titan's atmosphere. ${ }^{11,13}$

\section{Conclusions}

The main reaction products of the phenyl + acrylonitrile reaction are found to be Z-3phenylacrylonitrile [P1], E-3-phenylacrylonitrile [P2], ortho-cyanostyrene and 2-phenyl acrylonitrile. Among them, the two constitutional isomers [P1] and [P2] are found to have the 
lowest rate limiting barrier to formation. Quinoline has an overall rate limiting barrier well above the entrance channel of the reaction and is not detected. This can be further understood by looking at the competing pathways that are encountered in the formation of quinoline. Numerous intermediates, and among them also the assigned 2-cyano-1-phenylethyl radical (see red curve in Figure 3), have H-loss channels leading to E-3-phenylacrylonitrile and/or Z-3-phenylacrylonitrile that efficiently compete with the pathway leading to quinoline.

The ms-TPE spectrum the $\mathrm{m} / \mathrm{z} 130$ product does not show contributions from the direct phenyl + acrylonitrile adduct complexes (AC1-AC3), but rather a combination of already isomerized radical species $\mathbf{A}, \mathbf{B}$ and $\mathbf{C}$ which are potentially only one hydrogen atom loss away from the observed reaction products $\mathbf{P 1 - P 4}$. Thermalization may cause these species to be trapped under the lower temperature conditions, while they likely proceed to products when hot.

The lowest energy pathway to quinoline identified here is different from the lowest energy pathway to naphthalene with exclusively submerged transition states with respect to the reactants reported in the isoelectronic phenyl + vinylacetylene reaction. ${ }^{21}$ The analogous pathway to quinoline is found to be limited by a hydrogen migration barrier at $65.1 \mathrm{~kJ} / \mathrm{mol}$. We propose that the extraordinary stability of the nitrile group and its resistance to saturation prohibits this pathway. Although a lower-lying pathway was found, it still proceeds over a sizeable barrier and, as a consequence, ring formation in the phenyl + acrylonitrile reaction is kinetically controlled and is found not to be directly responsible for the production of PANHs.

Although the phenyl + acrylonitrile reaction does not yield quinoline directly, the formed products can still be important precursors for PANHs. Particularly, the intermediate open shell species of $\mathrm{C}_{9} \mathrm{H}_{8} \mathrm{~N}$ composition may play an important role as these are likely in the form of resonantly stabilized radicals (RSR). Subsequent reactions of these RSR may lead to polycyclic 
(aromatic) hydrocarbons in combustion environments. ${ }^{29,57}$ Furthermore, similar to what was suggested for the isoelectronic phenyl $+\mathrm{C}_{4} \mathrm{H}_{4}$ system, subsequent reactions of the products may lead to closing of the second ring, thus providing additional pathways to PANHs. ${ }^{20,58}$ However, the large stability of the nitrile group means that more saturated nitrogen-containing species are more likely to participate in ring formation processes. The avenues to the products $\mathbf{P 1}, \mathbf{P 2}, \mathbf{P 3}$ and $\mathbf{P 4}$ are de facto barrierless and are therefore expected to contribute to efficient molecular size growth in low temperature environments, such as the interstellar medium or Titan's atmosphere.

\section{Acknowledgement}

JB acknowledges the Netherlands Organisation for Scientific Research (Nederlandse Organisatie voor Wetenschappelijk Onderzoek, NWO) for a VIDI grant (grant number 723.016.006). JB acknowledges the European Union (EU) and Horizon 2020 funding awarded under the Marie Skłodowska-Curie action to the EUROPAH consortium, grant number 722346. This work was sponsored by NWO Exact and Natural Sciences for the use of supercomputer facilities (grant number 16638). Measurements have been performed at the VUV beamline of the Swiss Light Source, located at Paul Scherrer Institute (Villigen, Switzerland). P.H and A.B. acknowledge funding by the Swiss Federal Office of Energy (SI/501269-01) and Patrick Ascher for technical support. 


\section{References}

1. A. Leger and J. L. Puget, Astron. Astrophys., 1984, 137, L5-L8.

2. $\quad$ L. J. Allamandola, A. G. G. M. Tielens and J. R. Barker, Astrophys. J., 1985, 290, L25L28.

3. D. M. Hudgins, Bauschlicher, C.W and L. J. Allamandola, Astrophys. J., 2005, 632, 316.

4. $\quad$ A. Tielens, in Annu. Rev. Astron. Astr., Annual Reviews, Palo Alto, 2008, vol. 46, pp. 289337.

5. I. S. Kozin, O. F. A. Larsen, P. deVoogt, C. Gooijer and N. H. Velthorst, Anal. Chim. Acta, 1997, 354, 181-187.

6. H. Y. Chen and M. R. Preston, Environ. Sci. Technol., 1998, 32, 577-583.

7. E. A. J. Bleeker, S. Wiegman, P. de Voogt, M. Kraak, H. A. Leslie, E. de Haas and W. Admiraal, in Reviews of Environmental Contamination and Toxicology, ed. G. W. Ware, Springer, New York, 2002, vol. 173, pp. 39-83.

8. J. H. Waite, D. T. Young, T. E. Cravens, A. J. Coates, F. J. Crary, B. Magee and J. Westlake, Science, 2007, 316, 870-875.

9. V. Vuitton, R. V. Yelle and P. Lavvas, Philosophical Transactions of the Royal Society of London A: Mathematical, Physical and Engineering Sciences, 2009, 367, 729-741.

10. M. Lopez-Puertas, B. M. Dinelli, A. Adriani, B. Funke, M. Garcia-Comas, M. L. Moriconi, E. D'Aversa, C. Boersma and L. J. Allamandola, Astrophys. J., 2013, 770.

11. V. Vuitton, R. V. Yelle and M. J. McEwan, Icarus, 2007, 191, 722-742.

12. P. P. Lavvas, A. Coustenis and I. M. Vardavas, Planet. Space Sci., 2008, 56, 27-66.

13. V. A. Krasnopolsky, Icarus, 2009, 201, 226-256.

14. M. Frenklach, D. W. Clary, W. C. Gardiner Jr and S. E. Stein, Symp. (Int.) Combust., [Proc.], 1985, 20, 887-901.

15. M. Frenklach and E. D. Feigelson, Astrophys. J., 1989, 341, 372-384.

16. D. S. N. Parker, R. I. Kaiser, T. P. Troy and M. Ahmed, Angew. Chem., Int. Ed., 2014, 53, 7740-7744.

17. D. S. N. Parker, R. I. Kaiser, B. Bandyopadhyay, O. Kostko, T. P. Troy and M. Ahmed, Angew. Chem., Int. Ed., 2015, 54, 5421-5424.

18. R. I. Kaiser, D. S. N. Parker and A. M. Mebel, in Annual Review of Physical Chemistry, Vol 66, eds. M. A. Johnson and T. J. Martinez, 2015, vol. 66, pp. 43-67.

19. L. Zhao, R. I. Kaiser, B. Xu, U. Ablikim, M. Ahmed, D. Joshi, G. Veber, F. R. Fischer and A. M. Mebel, Nature Astronomy, 2018, DOI: 10.1038/s41550-018-0399-y.

20. N. W. Moriarty and M. Frenklach, Proc. Combust. Inst., 2000, 28, 2563-2568.

21. D. S. N. Parker, F. T. Zhang, Y. S. Kim, R. I. Kaiser, A. Landera, V. V. Kislov, A. M. Mebel and A. G. G. M. Tielens, Proc. Natl. Acad. Sci., 2012, 109, 53-58.

22. T. Yang, R. I. Kaiser, T. P. Troy, B. Xu, O. Kostko, M. Ahmed, A. M. Mebel, M. V. Zagidullin and V. N. Azyazov, Angew. Chem. Int. Ed., 2017, 56, 4515-4519.

23. L. Zhao, R. I. Kaiser, B. Xu, U. Ablikim, M. Ahmed, M. V. Zagidullin, V. N. Azyazov, A. H. Howlader, S. F. Wnuk and A. M. Mebel, The Journal of Physical Chemistry Letters, 2018, 9, 2620-2626.

24. D. S. N. Parker, R. I. Kaiser, O. Kostko, T. P. Troy, M. Ahmed, A. M. Mebel and A. G. G. M. Tielens, The Astrophysical Journal, 2015, 803, 53.

25. D. S. N. Parker and R. I. Kaiser, Chem. Soc. Rev., 2017, 46, 452-463. 
26. A. Bodi, P. Hemberger, D. L. Osborn and B. Sztaray, J. Phys. Chem. Lett., 2013, 4, 29482952.

27. T. Baer and R. P. Tuckett, Phys. Chem. Chem. Phys., 2017, DOI: 10.1039/C7CP00144D.

28. M. Steglich, V. B. F. Custodis, A. J. Trevitt, G. daSilva, A. Bodi and P. Hemberger, J. Am. Chem. Soc., 2017, 139, 14348-14351.

29. J. Bouwman, A. Bodi, J. Oomens and P. Hemberger, Phys. Chem. Chem. Phys., 2015, 17, 20508-20514.

30. I. M. Pastoors Johan, A. Bodi, P. Hemberger and J. Bouwman, Chem. Eur. J., 2017, 23, 13131-13140.

31. P. Hemberger, V. B. F. Custodis, A. Bodi, T. Gerber and J. A. van Bokhoven, Nature Communications, 2017, 8, 15946.

32. S. Liang, P. Hemberger, N. M. Neisius, A. Bodi, H. Grützmacher, J. Levalois-Grützmacher and S. Gaan, Chem. Eur. J., 2015, 21, 1073-1080.

33. A. Bodi, M. Johnson, T. Gerber, Z. Gengeliczki, B. Sztaray and T. Baer, Rev. Sci. Instrum., 2009, 80, 034101.

34. M. Johnson, A. Bodi, L. Schulz and T. Gerber, Nucl. Instrum. Meth. A., 2009, 610, 597603.

35. B. Sztáray, K. Voronova, K. G. Torma, K. J. Covert, A. Bodi, P. Hemberger, T. Gerber and D. L. Osborn, J. Chem. Phys., 2017, 147, 013944.

36. A. Golan, M. Ahmed, A. M. Mebel and R. I. Kaiser, Phys. Chem. Chem. Phys., 2013, 15, 341-347.

37. Q. Guan, K. N. Urness, T. K. Ormond, D. E. David, G. B. Ellison and J. W. Daily, Int. Rev. Phys. Chem., 2014, 33, 447-487.

38. A. Bodi, B. Sztáray, T. Baer, M. Johnson and T. Gerber, Rev. Sci. Instrum., 2007, 78, 084102.

39. B. Sztaray and T. Baer, Rev. Sci. Instrum., 2003, 74, 3763-3768.

40. A. Bodi and P. Hemberger, Phys. Chem. Chem. Phys., 2014, 16, 505-515.

41. M. J. Frisch, G. W. Trucks, H. B. Schlegel, G. E. Scuseria, M. A. Robb, J. R. Cheeseman, G. Scalmani, V. Barone, B. Mennucci, G. A. Petersson, H. Nakatsuji, M. Caricato, X. Li, H. P. Hratchian, A. F. Izmaylov, J. Bloino, G. Zheng, J. L. Sonnenberg, M. Hada, M. Ehara, K. Toyota, R. Fukuda, J. Hasegawa, M. Ishida, T. Nakajima, Y. Honda, O. Kitao, H. Nakai, T. Vreven, J. J. A. Montgomery, J. E. Peralta, F. Ogliaro and J. J. H. M. Bearpark, E. Brothers, K. N. Kudin, V. N. Staroverov, R. Kobayashi, J. Normand, K. Raghavachari, A. Rendell, J. C. Burant, S. S. Iyengar, J. Tomasi, M. Cossi, N. Rega, J. M. Millam, M. Klene, J. E. Knox, J. B. Cross, V. Bakken, C. Adamo, J. Jaramillo, R. Gomperts, R. E. Stratmann, O. Yazyev, A. J. Austin, R. Cammi, C. Pomelli, J. W. Ochterski, R. L. Martin, K. Morokuma, V. G. Zakrzewski, G. A. Voth, P. Salvador, J. J. Dannenberg, S. Dapprich, A. D. Daniels, Ö. Farkas, J. B. Foresman, J. V. Ortiz, J. Cioslowski, and D. J. Fox Gaussian, Inc., 2009, Wallingford CT.

42. J. A. Montgomery, M. J. Frisch, J. W. Ochterski and G. A. Petersson, J. Chem. Phys., 1999, 110, 2822-2827.

43. J. A. Montgomery, M. J. Frisch, J. W. Ochterski and G. A. Petersson, J. Chem. Phys., 2000, 112, 6532-6542.

44. A. Mozhayskiy and A. I. Krylov, ezSpectrum, Version 3.0.

45. P. Oßwald, P. Hemberger, T. Bierkandt, E. Akyildiz, M. Köhler, A. Bodi, T. Gerber and T. Kasper, Rev. Sci. Instrum., 2014, 85, 025101. 
46. E. Reusch, F. Holzmeier, P. Constantinidis, P. Hemberger and I. Fischer, Angew. Chem. Int. Ed., 2017, 56, 8000-8003.

47. B. Sztaray, A. Bodi and T. Baer, J. Mass Spectrom., 2010, 45, 1233-1245.

48. M. Sander, L. A. Chewter, K. Mullerdethlefs and E. W. Schlag, Physical Review A, 1987, 36, 4543-4546.

49. K. Ohno, S. Matsumoto, K. Imai and Y. Harada, J. Phys. Chem., 1984, 88, 206-209.

50. $\quad$ R. Egdell, J. C. Green and C. N. R. Rao, Chem. Phys. Lett., 1975, 33, 600-607.

51. V. Butcher, M. L. Costa, J. M. Dyke, A. R. Ellis and A. Morris, Chem. Phys., 1987, 115, 261-267.

52. G. I. Nemeth, H. L. Selzle and E. W. Schlag, Chem. Phys. Lett., 1993, 215, 151-155.

53. C. H. Kwon, H. L. Kim and M. S. Kim, J. Phys. Chem. A, 2003, 107, 10969-10975.

54. M. J. S. Dewar and S. D. Worley, J. Chem. Phys., 1969, 50, 654-667.

55. J. Bouwman, B. Sztáray, J. Oomens, P. Hemberger and A. Bodi, J. Phys. Chem. A, 2015, 119, 1127-1136.

56. J. H. D. Eland and C. J. Danby, Z. Naturforsch. A, 1968, 23, 355.

57. N. Hansen, J. A. Miller, S. J. Klippenstein, P. R. Westmoreland and K. Kohse-Hoinghaus, Combust., Explos. Shock Waves, 2012, 48, 508-515.

58. A. M. Mebel, A. Landera and R. I. Kaiser, J. Phys. Chem. A, 2017, 121, 901-926. 